\title{
Spot sign as a predictor of rebleeding after endoscopic surgery for intracerebral hemorrhage
}

\author{
Koichi Miki, MD, ${ }^{1}$ Kenji Yagi, MD, PhD, ${ }^{1}$ Masani Nonaka, MD, PhD, ${ }^{1}$ Mitsutoshi Iwaasa, MD, PhD, ${ }^{2}$ \\ Hiroshi Abe, MD, PhD, ${ }^{1}$ Takashi Morishita, MD, PhD, ${ }^{1}$ Hisatomi Arima, MD, PhD, ${ }^{3}$ and \\ Tooru Inoue, MD, PhD'
}

${ }^{1}$ Department of Neurosurgery, ${ }^{2}$ Department of Emergency and Critical Care, and ${ }^{3}$ Department of Preventive Medicine and Public Health, Faculty of Medicine, Fukuoka University Hospital and School of Medicine, Fukuoka University, Fukuoka, Japan

\begin{abstract}
OBJECTIVE In patients with spontaneous intracerebral hemorrhage $(\mathrm{sICH})$, postoperative recurrent hemorrhage (PRH) is one of the most severe complications after endoscopic evacuation of hematoma (EEH). However, no predictors of this complication have been identified. In the present study, the authors retrospectively investigated whether PRH can be preoperatively predicted by the presence of the spot sign on CT scans.

METHODS In total, 143 patients with sICH were treated by EEH between June 2009 and March 2017, and 127 patients who underwent preoperative CT angiography were included in this study. Significant correlations of PRH with the patients' baseline, clinical, and radiographic characteristics, including the spot sign, were evaluated using multivariable logistic regression models.
\end{abstract}

RESULTS The incidence of and risk factors for PRH were assessed in 127 patients with available data. PRH occurred in $9(7.1 \%)$ patients. Five $(21.7 \%)$ cases of PRH were observed among 23 patients with the spot sign, whereas only 4 (3.8\%) cases of PRH occurred among 104 patients without the spot sign. The spot sign was the only independent predictor of PRH (OR 5.81, 95\% Cl 1.26-26.88; $p=0.02$ ). The following factors were not independently associated with PRH: age, hypertension, poor consciousness, antihemostatic factors (thrombocytopenia, coagulopathy, and use of antithrombotic drugs), the location and size of the $\mathrm{s} \mathrm{CH}$, other radiographic findings (black hole sign and blend sign), surgical duration and procedures, and early surgery.

CONCLUSIONS The spot sign is likely to be a strong predictor of $\mathrm{PRH}$ after $\mathrm{EEH}$ among patients with sICH. Complete and careful control of bleeding in the operative field should be ensured when surgically treating such patients. New surgical strategies and procedures might be needed to improve these patients' outcomes.

https://thejns.org/doi/abs/10.3171/2017.12.JNS172335

KEYWORDS intracerebral hemorrhage; computed tomography angiography; surgery; spot sign; recurrent hemorrhage; vascular disorders

$\mathrm{S}$ PONTANEOUS intracerebral hemorrhage (sICH) is one of the most devastating forms of cerebrovascular disease. $\mathrm{sICH}$ accounts for approximately $15 \%$ of all strokes in the US and Europe and 20\%-30\% of strokes in Asian populations. ${ }^{20}$ Surgical treatment may prevent secondary neuronal injury due to formation of hematomas and perihematoma edema, which result in a poor prognosis, although surgery via craniotomy has failed to improve patient outcomes in several randomized trials. ${ }^{24}$ The effectiveness of surgery is controversial. ${ }^{4,24}$ Reduction in

ABBREVIATIONS EEH = endoscopic evacuation of hematoma; GCS = Glasgow Coma Scale; IQR = interquartile range; IVH = intraventricular hematoma; $\mathrm{PRH}=$ postoperative recurrent hemorrhage; $\mathrm{SICH}=$ spontaneous intracerebral hemorrhage.

SUBMITTED September 18, 2017. ACCEPTED December 11, 2017.

INCLUDE WHEN CITING Published online May 25, 2018; DOI: 10.3171/2017.12.JNS172335. 
surgical complications or the performance of less invasive surgery is considered to improve the patients' prognosis.

Minimally invasive endoscopic surgery has recently been applied to treat patients with sICH and is expected to improve patients' prognosis. ${ }^{2,13,26}$ Although postoperative recurrent hemorrhage (PRH) is one of the most severe complications after endoscopic evacuation of hematoma (EEH), the risk factors for this complication have not been identified. The spot sign on CT is considered to be a significant predictor of spontaneous expansion of sICH and has been reported as a risk factor for PRH after craniotomy. ${ }^{4,9,25}$ However, the relationship of the spot sign with PRH after EEH is unknown. In the present study, we investigated whether the spot sign predicts PRH after EEH., 20,24

\section{Methods}

In this retrospective cohort study, we investigated the relationship between the spot sign and PRH after EEH. The study population comprised patients who underwent endoscopic surgery to evacuate parenchymal sICH and in whom preoperative $\mathrm{CT}$ angiography was performed. sICH was defined as hemorrhage not associated with traumatic brain injury, aneurysms, arteriovenous malformations, arteriovenous fistulas, moyamoya disease, or tumors. The protocol for this retrospective study was approved by the ethics committee at our institution, and the board waived the need for patient consent. In total, 164 patients with sICH were treated by endoscopic surgery at our institution between June 2009 and March 2017. Of these, 143 patients had significant parenchymal sICH and were treated by $\mathrm{EEH}$. Twenty-one patients with a massive intraventricular hematoma (IVH) in whom the endoscopic surgery was applied only for treatment of the IVH and not for treatment of the parenchymal hematoma were excluded from the study. Most of the patients underwent routine CT angiography to rule out secondary hemorrhage before the surgery. Of the 143 patients, 127 underwent preoperative CT angiography, and the data were available to assess the presence of the spot sign. These patients comprised the study population (Fig. 1). The other 16 patients were excluded because CT angiography was not performed due to renal dysfunction, contrast agent allergy, or urgent surgery with no time for the examination. The patients' baseline and clinical characteristics and radiographic findings are shown in Table 1.

The surgical indications were as follows: 1) sICH with a significant mass effect and neurological deterioration, 2) a basal ganglia hematoma of $>10 \mathrm{ml}$ and associated with a massive IVH and hydrocephalus, and 3) a cerebellar hematoma of $>3 \mathrm{~cm}$ in diameter. sICH was evacuated via a transcortical or transventricular approach. EEH was performed via a burr hole or small craniotomy under general anesthesia. In patients with sICH that was associated with significant acute hydrocephalus or a massive IVH, the IVH was simultaneously removed endoscopically with evacuation of the parenchymal hematoma. EEH performed within 8 hours posthemorrhage was defined as early surgery. If information regarding the volume and location of the residual hematoma was not obtained during the surgery, the surgeon stopped the procedure and performed a second $\mathrm{EEH}$ after confirming the volume and location. The treat-

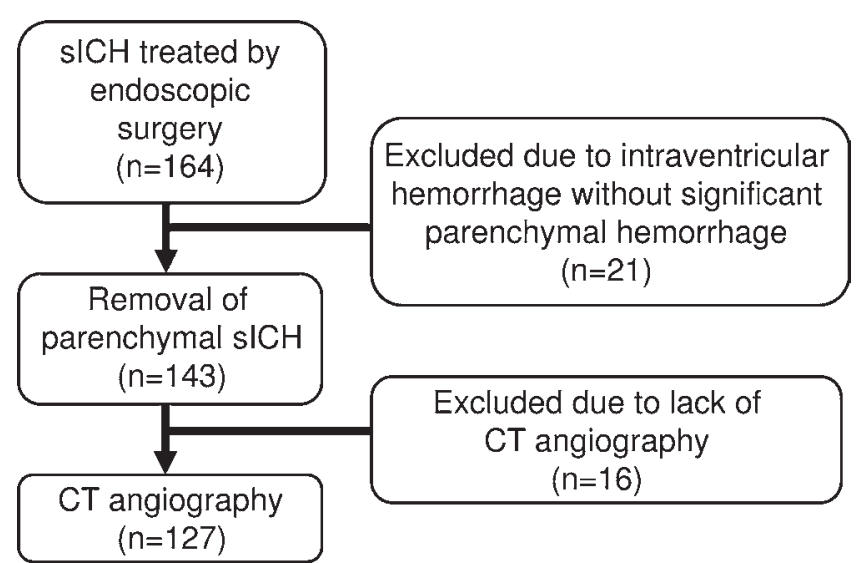

FIG. 1. Flowchart of inclusion and exclusion criteria for patients with $\mathrm{SICH}$.

ment was performed as a staged surgery. Active bleeding was defined as intraoperative hemorrhage from a vessel requiring electrosurgical coagulation. The surgeons were recorded as Surgeons A, B, C, and others. The details of the surgical procedures are shown in the Online Supplement.

A CT scan was performed before the EEH $(132 \pm 121$ minutes before surgery). The hematoma volume and location were recorded. sICH was classified according to presence of the black hole sign or blend sign on the CT scan (details are shown in the Online Supplement). Briefly, the black hole sign is defined as a hypoattenuated round area encapsulated within the hyperattenuating hematoma. ${ }^{15}$ The blend sign is defined as blending of a hypoattenuating area with an adjacent hypoattenuating region. ${ }^{14}$

CT angiography was also performed $143 \pm 239$ minutes before the EEH by using a bolus-tracking method in which $80-100 \mathrm{ml}$ of nonionic iodinated contrast was injected. The presence of the spot sign on CT angiography images was recorded. Briefly, the spot sign was defined as a hyperdense spot within a hematoma without connection to an outside vessel. These details are also shown in the Online Supplement.

The patients routinely underwent repeat CT scans immediately after, 12-36 hours after, and 3-7 days after the surgery. They also underwent a CT scan when their neurological state deteriorated remarkably. The occurrence of PRH was recorded during the first 3 days following the surgery. PRH was defined as a different appearance of sICH compared with the preoperative appearance on the CT scan, and a mass effect causing neurological deterioration. A residual hematoma was distinguished from PRH based on the intraoperative record and consistency of the sICH appearance between the preoperative and postoperative CT scans (i.e., the same shape and density) and no trace of having performed the endoscopic procedure, such as the presence of air or water bubbles. All radiographic evaluations of the preoperative and postoperative CT scans were independently performed by 2 experienced neurosurgeons in a blinded manner. When the assessments differed, interrater agreement was achieved by discussion.

Preoperative thrombocytopenia was defined as a platelet count of $<100 \times 10^{3} / \mu l$. Coagulopathy was defined as 
TABLE 1. Baseline, clinical, and radiographic characteristics in patients with sICH

\begin{tabular}{lc}
\hline \multicolumn{1}{c}{ Characteristic } & Patients w/ EEH \\
\hline No. of patients & 127 \\
\hline Age in yrs & $66.6 \pm 11.5$ \\
\hline Female sex & $51(40.2)$ \\
\hline Hypertension & $80(63.0)$ \\
\hline Diabetes mellitus & $24(18.9)$ \\
\hline Smoking & $48(37.8)$ \\
\hline Daily alcohol consumption & $45(35.4)$ \\
\hline Thrombocytopenia & $8(6.3)$ \\
\hline Coagulopathy & $6(4.7)$ \\
\hline Antiplatelet therapy & $24(18.9)$ \\
\hline Anticoagulant therapy & $11(8.7)$ \\
\hline Median GCS score, IQR & $10,7-12$ \\
\hline Hematoma vol (ml) & $51.1 \pm 36.9$ \\
\hline Location & \\
\hline Basal ganglia & $92(72.4)$ \\
\hline Lobar & $16(12.6)$ \\
\hline Cerebellum & $19(15.0)$ \\
\hline Radiographic findings & \\
\hline Black hole sign & $15(11.8)$ \\
\hline Blend sign & $11(8.7)$ \\
\hline Spot sign & $23(18.1)$ \\
\hline
\end{tabular}

Data are presented as the mean $\pm S D$, number $(\%)$, or median and IQR (25th75th percentile).

an activated partial thromboplastin time of $\geq 40$ seconds or a prothrombin time-international normalized ratio of $>$ 1.4. Patients undergoing oral antiplatelet or anticoagulant therapy were recorded. Anticoagulant therapy involved the use of warfarin $(n=11)$. Ten of these patients underwent preoperative administration of prothrombin complex concentrates or vitamin $\mathrm{K}$ to neutralize the effect of the anticoagulants, whereas 1 was not given such medication because of a normal activated partial thromboplastin time and prothrombin time-international normalized ratio. The definitions of the patients' other background factors are shown in the Online Supplement.

Statistical analyses were performed with SPSS version 24 software (IBM Corp.). Continuous variables are expressed as the mean $\pm \mathrm{SD}$ or median and interquartile range ([IQR], 25th-75th percentile), and categorical variables are expressed as number (percentage). Predictors of PRH were first assessed using a univariable logistic regression model, and age and possible predictors with a p value of $<0.20$ were then included in the multivariable logistic regression analyses for assessment of independent predictors. A $p$ value of $<0.05$ was considered statistically significant.

\section{Results}

Of the 127 patients, $9(7.1 \%)$ died (6 died directly of sICH-induced brain damage, and 3 died of other causes, such as mesenteric artery occlusion or remote cerebral infarction). The parenchymal hematoma was mostly removed (> 80\% of its volume) in $107(84.3 \%)$ patients.

Of the 127 patients, 9 (7.1\%) developed PRH, and it occurred within 1 day after EEH. However, no other hemorrhagic complications occurred, such as a remote ICH or acute subdural or epidural hematoma. PRH was associated with a high mortality rate of $66.7 \%$; 5 patients died directly of PRH, and 1 patient died of a mesenteric artery occlusion.

Five $(21.7 \%)$ cases of PRH were observed among 23 patients with the spot sign, whereas only 4 (3.8\%) cases of PRH were observed among 104 patients without the spot sign. The baseline and clinical characteristics and radiographic findings were compared between the patients with and without PRH (Table 2). Only an increased surgical duration (OR 1.68, 95\% CI 1.07-2.63; $\mathrm{p}=0.03)$ and presence of the spot sign (OR 6.94, 95\% CI 1.70-28.37; $\mathrm{p}=0.007$ ) were significant risk factors in the univariate regression analyses. Next, a multivariate analysis involving age and all variables with a $\mathrm{p}$ value of $<0.20$ in the univariate analyses was performed. The spot sign was the only independent predictor of PRH (OR 5.81, 95\% CI 1.26-26.88; $\mathrm{p}=0.02$ ).

The association of the spot sign with intraoperative active bleeding was evaluated. The spot sign was a significant risk factor for intraoperative active bleeding (OR $3.64,95 \%$ CI 1.43-9.28; $p=0.007)$. The association of the spot sign with early surgery was also assessed. Although our surgical indication did not consider the presence versus absence of the spot sign, we found that the spot sign was significantly associated with early surgery (OR 11.16, 95\% CI 1.44-86.24; $\mathrm{p}=0.02$ ).

\section{Discussion}

Endoscopic surgery for sICH is reportedly associated with less invasion, a shorter operative duration, a higher hematoma evacuation rate, a lower frequency of complications, and a better prognosis than traditional craniotomy. ${ }^{6,13,26} \mathrm{PRH}$ reportedly occurs in up to $9.5 \%$ of patients after EEH; ${ }^{2,13,26}$ this is consistent with the findings of our study. The risk factors for PRH in patients undergoing endoscopic surgery are not well understood, but we have herein demonstrated that the spot sign is an independent risk factor for PRH after EEH.

In previous studies, the spot sign on $\mathrm{CT}$ was identified at admission in $30 \%-37 \%$ of patients with sICH., . $^{4,10,25}$ It is considered a significant predictor of spontaneous expansion of sICH, with a high sensitivity of $51 \%-91 \%$ and specificity of $85 \%-89 \%$ under conservative treatment. ${ }^{9,25}$ Moreover, the spot sign was recently reported as an independent risk factor of PRH after craniotomy for $\mathrm{sICH}^{4}$ Several risk factors are reportedly associated with the spot sign: a large hematoma volume, anticoagulation, early presentation, a low Glasgow Coma Scale (GCS) score on presentation, a mean arterial blood pressure of $>120$ $\mathrm{mm} \mathrm{Hg}$, the presence of IVH, and the APOE \&2 allele. ${ }^{3}$ Recent studies using dynamic CT angiography have suggested that the spot sign signifies active extravasation in the hematoma. ${ }^{710}$ Extravasation of contrast medium may be attributed to continuous bleeding or a pseudoaneurysm 
TABLE 2. Effects of predictors of rebleeding after endoscopic surgery in patients with sICH

\begin{tabular}{|c|c|c|c|c|c|c|}
\hline \multirow[b]{2}{*}{ Predictor } & \multicolumn{3}{|c|}{ Crude Model } & \multicolumn{3}{|c|}{ Multivariable Model } \\
\hline & OR & $95 \% \mathrm{Cl}$ & p Value & OR & $95 \% \mathrm{Cl}$ & p Value \\
\hline Age (per 10-yr increase) & 0.87 & $0.49-1.55$ & 0.63 & 0.71 & $0.35-1.46$ & 0.35 \\
\hline Female sex & 1.21 & $0.31-4.73$ & 0.79 & & & \\
\hline Hypertension & 0.72 & $0.18-2.81$ & 0.63 & & & \\
\hline Diabetes mellitus & 0.52 & $0.06-4.34$ & 0.67 & & & \\
\hline Smoking & 1.35 & $0.34-5.28$ & 0.67 & & & \\
\hline Daily alcohol consumption & 0.91 & $0.22-3.80$ & 0.89 & & & \\
\hline Thrombocytopenia & 1.98 & $0.22-18.15$ & 0.55 & & & \\
\hline Coagulopathy & \multicolumn{2}{|c|}{ Not calculable } & & & & \\
\hline Antiplatelet drug & 1.25 & $0.24-6.42$ & 0.79 & & & \\
\hline Anticoagulant drug & \multicolumn{2}{|c|}{ Not calculable } & & & & \\
\hline GCS score $<9$ & 3.36 & $0.80-14.13$ & 0.10 & 3.17 & $0.61-16.57$ & 0.17 \\
\hline Hematoma vol (per 5-ml increase) & 1.03 & $0.96-1.11$ & 0.44 & & & \\
\hline \multicolumn{7}{|l|}{ Location } \\
\hline Basal ganglia & Ref & & & & & \\
\hline Lobar & 0.81 & $0.09-7.06$ & 0.85 & & & \\
\hline Cerebellum & 0.68 & $0.08-5.83$ & 0.72 & & & \\
\hline \multicolumn{7}{|l|}{ Radiographic findings } \\
\hline Black hole sign & 0.93 & $0.11-7.99$ & 0.95 & & & \\
\hline Blend sign & 1.35 & $0.15-11.91$ & 0.79 & & & \\
\hline Spot sign & 6.94 & $1.70-28.37$ & 0.007 & 5.81 & $1.26-26.88$ & 0.02 \\
\hline Early surgery & 3.37 & $0.41-27.99$ & 0.26 & & & \\
\hline Duration of surgery (per 1-hr increase) & 1.68 & $1.07-2.63$ & 0.03 & 1.39 & $0.81-2.39$ & 0.24 \\
\hline Transventricular approach & 2.83 & $0.71-11.31$ & 0.14 & 1.31 & $0.19-9.00$ & 0.78 \\
\hline Removal of IVH & 3.24 & $0.77-13.62$ & 0.11 & 1.05 & $0.13-8.89$ & 0.96 \\
\hline Staged surgery & \multicolumn{2}{|c|}{ Not calculable } & & & & \\
\hline \multicolumn{7}{|l|}{ Surgeon } \\
\hline A & Ref & & & & & \\
\hline$B$ & 1.30 & $0.17-10.05$ & 0.80 & & & \\
\hline$C$ & 1.24 & $0.16-9.55$ & 0.84 & & & \\
\hline Others & 0.77 & $0.12-4.87$ & 0.78 & & & \\
\hline
\end{tabular}

at an injured small vessel. Consistent with a previous study of craniotomy for sICH, the present study showed that the spot sign was a significant risk factor for intraoperative active bleeding. ${ }^{4}$ We consider that the fragile vessel illustrated by the spot sign contributed to PRH as well as spontaneous hematoma expansion under conservative treatment, although the location of the spot sign was not accurately identified intraoperatively, and the active bleeding might not have been associated with a spot sign-related vessel.

An antithrombotic state such as that induced by thrombocytopenia, coagulopathy, and the use of antiplatelet and anticoagulant agents has been reported to increase the risk of intracranial hemorrhagic complications after craniotomy for sICH, tumors, aneurysms, and trauma. ${ }^{1,521-23} \mathrm{He}-$ mostasis is considered important to help prevent PRH. In the present study, however, factors associated with an antithrombotic state were not risk factors for PRH, although a shortage of power in the analyses may have contributed to this lack of an association. In addition, most of the patients undergoing anticoagulant therapy were preoperatively given vitamin $\mathrm{K}$ or prothrombin complex, which may also have contributed to the suppression of PRH by neutralizing the hemorrhagic effect of anticoagulant therapy.

The optimal timing of surgery for sICH has not been established. In a meta-analysis, early surgery within 8 hours posthemorrhage reportedly improved the patients' prognosis. ${ }^{11}$ Surgical evacuation of sICH might suppress secondary brain injury by improving regional blood flow and restricting the release of toxic breakdown products by the clot. ${ }^{12}$ In contrast, early surgery within 4 hours posthemorrhage is a reported risk factor for $\mathrm{PRH} .{ }^{17}$ In the present study, early surgery ( $<8$ hours from onset) was not associated with PRH. Further studies are needed to establish the best timing of EEH.

A treatment strategy for spot sign-positive sICH with a high risk of expansion has not been established. Urgent surgery may prevent spontaneous expansion of sICH by treating the point of vessel rupture illustrated by the spot 
sign in patients with a high risk of sICH expansion. In addition, spot sign-positive sICH tends to be larger in size and theoretically provides further rationale for decompression. ${ }^{4}$ However, the performance of surgery should be carefully considered because of the high risk of $\mathrm{PRH}$, which results in a poor outcome. We believe that the benefit of surgery outweighs the risk in patients with the spot sign. The reported in-hospital mortality rate among spot signpositive patients was $41 \%$ under conservative treatment in a previous large study. ${ }^{8}$ The in-hospital mortality rate was higher than $28 \%$ in patients who underwent craniotomy, ${ }^{4,8}$ and it was $7.1 \%$ in those who had endoscopic surgery in the present study.

When surgically treating sICH in patients with the spot sign, extra effort and new treatments are needed to prevent PRH. For this purpose, we suggest the following. Complete and careful control of bleeding in the operative field is important. Irrigating and inflating the hematoma cavity with saline or artificial CSF is a useful method of identifying bleeding points. ${ }^{19}$ Identifying a ruptured small vessel illustrated by the spot sign under the guidance of a navigation system and direct coagulation of the rupture point may suppress the development of PRH. Administration of coagulant factors also has the potential to inhibit PRH. In a trial of activated factor VII for ICH, recombinant activated factor VII suppressed hematoma expansion in patients undergoing conservative treatment. ${ }^{16}$ Close and careful postoperative neurological and radiographic monitoring is necessary in such patients. An early repeated CT scan is recommended to identify PRH for which reoperation is needed. Intensive control of blood pressure may suppress the incidence of PRH, although its effect did not inhibit spontaneous expansion of sICH in patients with the spot sign in a secondary analysis of the Antihypertensive Treatment of Acute Cerebral Hemorrhage II (ATACH-II) trial. ${ }^{18}$

This study has several limitations. The first is its retrospective, single-center design. Second, all patients were not included in the study because in some preoperative CT angiography examinations were lacking. Third, the surgeons may have been aware of the presence of the spot sign. In fact, patients with the spot sign more frequently underwent early surgery, although whether the spot sign was present or absent was not included in our surgical indication. Finally, the statistical power of this study is somewhat limited, although this is one of the largest studies to have investigated the association between the spot sign and PRH. A prospective multicenter study with a sufficient number of patients is needed to confirm our findings regarding the spot sign and PRH. Furthermore, a new treatment strategy should be established to improve the surgical outcomes in patients with unstable sICH with the spot sign.

\section{Conclusions}

The spot sign is likely to be a strong predictor of PRH after EEH among patients with sICH. When surgically treating sICH in patients with the spot sign, complete and careful control of bleeding in the operative field should be ensured. Furthermore, new surgical strategies and procedures might be needed to improve these patients' outcomes.

\section{Acknowledgments}

We thank Angela Morben, DVM, ELS, from Edanz Group for editing a draft of this manuscript.

\section{References}

1. Asher RA, Morgenstern DA, Moon LD, Fawcett JW: Chondroitin sulphate proteoglycans: inhibitory components of the glial scar. Prog Brain Res 132:611-619, 2001

2. Auer LM, Deinsberger W, Niederkorn K, Gell G, Kleinert R, Schneider G, et al: Endoscopic surgery versus medical treatment for spontaneous intracerebral hematoma: a randomized study. J Neurosurg 70:530-535, 1989

3. Brouwers HB, Goldstein JN, Romero JM, Rosand J: Clinical applications of the computed tomography angiography spot sign in acute intracerebral hemorrhage: a review. Stroke 43:3427-3432, 2012

4. Brouwers HB, Raffeld MR, van Nieuwenhuizen KM, Falcone GJ, Ayres AM, McNamara KA, et al: CT angiography spot sign in intracerebral hemorrhage predicts active bleeding during surgery. Neurology 83:883-889, 2014

5. Bullock R, Hanemann CO, Murray L, Teasdale GM: Recurrent hematomas following craniotomy for traumatic intracranial mass. J Neurosurg 72:9-14, 1990

6. Cho DY, Chen CC, Chang CS, Lee WY, Tso M: Endoscopic surgery for spontaneous basal ganglia hemorrhage: comparing endoscopic surgery, stereotactic aspiration, and craniotomy in noncomatose patients. Surg Neurol 65:547-556, 2006

7. d'Esterre CD, Chia TL, Jairath A, Lee TY, Symons SP, Aviv RI: Early rate of contrast extravasation in patients with intracerebral hemorrhage. AJNR Am J Neuroradiol 32:18791884,2011

8. Delgado Almandoz JE, Yoo AJ, Stone MJ, Schaefer PW, Oleinik A, Brouwers HB, et al: The spot sign score in primary intracerebral hemorrhage identifies patients at highest risk of in-hospital mortality and poor outcome among survivors. Stroke 41:54-60, 2010

9. Demchuk AM, Dowlatshahi D, Rodriguez-Luna D, Molina CA, Blas YS, Dzialowski I, et al: Prediction of haematoma growth and outcome in patients with intracerebral haemorrhage using the CT-angiography spot sign (PREDICT): a prospective observational study. Lancet Neurol 11:307-314, 2012

10. Dowlatshahi D, Wasserman JK, Momoli F, Petrcich W, Stotts G, Hogan M, et al: Evolution of computed tomography angiography spot sign is consistent with a site of active hemorrhage in acute intracerebral hemorrhage. Stroke 45:277-280, 2014

11. Gregson BA, Broderick JP, Auer LM, Batjer H, Chen XC, Juvela $S$, et al: Individual patient data subgroup meta-analysis of surgery for spontaneous supratentorial intracerebral hemorrhage. Stroke 43:1496-1504, 2012

12. Jüttler E, Steiner T: Treatment and prevention of spontaneous intracerebral hemorrhage: comparison of EUSI and AHA/ ASA recommendations. Expert Rev Neurother 7:14011416,2007

13. Kuo LT, Chen CM, Li CH, Tsai JC, Chiu HC, Liu LC, et al: Early endoscope-assisted hematoma evacuation in patients with supratentorial intracerebral hemorrhage: case selection, surgical technique, and long-term results. Neurosurg Focus 30(4):E9, 2011

14. Li Q, Zhang G, Huang YJ, Dong MX, Lv FJ, Wei X, et al: Blend sign on computed tomography: novel and reliable predictor for early hematoma growth in patients with intracerebral hemorrhage. Stroke 46:2119-2123, 2015

15. Li Q, Zhang G, Xiong X, Wang XC, Yang WS, Li KW, et al: Black hole sign: novel imaging marker that predicts hematoma growth in patients with intracerebral hemorrhage. Stroke 47:1777-1781, 2016 
16. Mayer SA, Brun NC, Begtrup K, Broderick J, Davis S, Diringer MN, et al: Recombinant activated factor VII for acute intracerebral hemorrhage. N Engl J Med 352:777-785, 2005

17. Morgenstern LB, Demchuk AM, Kim DH, Frankowski RF, Grotta JC: Rebleeding leads to poor outcome in ultraearly craniotomy for intracerebral hemorrhage. Neurology 56:1294-1299, 2001

18. Morotti A, Brouwers HB, Romero JM, Jessel MJ, Vashkevich A, Schwab K, et al: Intensive blood pressure reduction and spot sign in intracerebral hemorrhage: a secondary analysis of a randomized clinical trial. JAMA Neurol 74:950-960, 2017

19. Nagasaka T, Inao S, Ikeda H, Tsugeno M, Okamoto T: Inflation-deflation method for endoscopic evacuation of intracerebral haematoma. Acta Neurochir (Wien) 150:685-690, 2008

20. Nagasaka T, Inao S, Wakabayashi T: What does the CT angiography "spot sign" of intracerebral hemorrhage mean in modern neurosurgical settings with minimally invasive endoscopic techniques? Neurosurg Rev 36:341-348, 2013

21. Palmer JD, Sparrow OC, Iannotti F: Postoperative hematoma: a 5-year survey and identification of avoidable risk factors. Neurosurgery 35:1061-1065, 1994

22. Seifman MA, Lewis PM, Rosenfeld JV, Hwang PY: Postoperative intracranial haemorrhage: a review. Neurosurg Rev 34:393-407, 2011

23. Umebayashi D, Mandai A, Osaka Y, Nakahara Y, Tenjin $\mathrm{H}$ : Effects and complications of stereotactic aspiration for spontaneous intracerebral hemorrhage. Neurol Med Chir (Tokyo) 50:538-544, 2010

24. Vespa PM, Martin N, Zuccarello M, Awad I, Hanley DF: Surgical trials in intracerebral hemorrhage. Stroke 44 (6 Suppl 1):S79-S82, 2013

25. Wada R, Aviv RI, Fox AJ, Sahlas DJ, Gladstone DJ, Tomlinson G, et al: CT angiography "spot sign" predicts hematoma expansion in acute intracerebral hemorrhage. Stroke 38:1257-1262, 2007

26. Xu X, Chen X, Li F, Zheng X, Wang Q, Sun G, et al: Effectiveness of endoscopic surgery for supratentorial hypertensive intracerebral hemorrhage: a comparison with craniotomy. $\mathbf{J}$ Neurosurg 128:553-559, 2018

\section{Disclosures}

This work was partly supported by the Japan Society for Promotion of Science (C15K10306). Dr. Yagi received grant support from the Japan Society for the Promotion of Science and AO Spine. Dr. Morishita received grant support from the Japan Society for the Promotion of Science, St. Luke Life Science Institute, Nakatomi Foundation, Takeda Science Foundation, Uehara Memorial Foundation, and Central Research Institute of Fukuoka University. He also received honoraria from Boston Scientific and Medtronic as a consultant within the past 12 months. Dr. Inoue received grant support from the Clinical Research Promotion Foundation Japan and Japan Agency for Medical Research and Development.

\section{Author Contributions}

Conception and design: Yagi. Acquisition of data: Yagi, Miki. Analysis and interpretation of data: Yagi, Iwaasa, Arima. Drafting the article: Yagi, Miki. Critically revising the article: Iwaasa, Abe, Morishita. Reviewed submitted version of manuscript: Nonaka. Approved the final version of the manuscript on behalf of all authors: Yagi. Statistical analysis: Arima. Administrative/techni$\mathrm{cal} /$ material support: Nonaka. Study supervision: Inoue.

\section{Supplemental Information Online-Only Content}

Supplemental material is available with the online version of the article.

Online Supplement. https://thejns.org/doi/suppl/10.3171/2017.

$$
\text { 12.JNS172335. }
$$

\section{Correspondence}

Kenji Yagi: Fukuoka University, Fukuoka, Japan. kenji-yagi@ mail.goo.ne.jp. 